\title{
Supergauge theories in aether superspace
}

\author{
A. C. Lehum, ${ }^{1,2, * *}$ J. R. Nascimento, ${ }^{3,}$ A. Yu. Petrov, ${ }^{3, t}$ and A. J. da Silva ${ }^{1, t}$ \\ ${ }^{1}$ Instituto de Física, Universidade de São Paulo \\ Caixa Postal 66318, 05315-970, São Paulo, SP, Brazil \\ ${ }^{2}$ Escola de Ciências e Tecnologia, Universidade Federal do Rio Grande do Norte \\ Caixa Postal 1524, 59072-970, Natal, Rio Grande do Norte, Brazil \\ ${ }^{3}$ Departamento de Física, Universidade Federal da Paraíba \\ Caixa Postal 5008, 58051-970, João Pessoa, Paraíba, Brazil
}

\begin{abstract}
Within the superfield method we extend the formulation of the Lorentz-breaking aether superspace for supergauge theories, both in three- and in four-dimensional cases.

PACS numbers: 11.30.Pb, 11.30.Cp
\end{abstract}

*Electronic address: andrelehum@ect.ufrn.br

${ }^{\dagger}$ Electronic address: jroberto,petrov@fisica.ufpb.br

${ }^{\ddagger}$ Electronic address: ajsilva@fma.if.usp.br 


\section{INTRODUCTION}

The idea of the Lorentz symmetry breaking has been intensively discussed during the last years (for a review, see f.e. [1]). The interest in this line of studies is motivated by the fact that the presence of the Lorentz-breaking additive modifications of field theory models, essentially enriches their structure [2]. At the same time, since supersymmetry is treated as a fundamental physical symmetry, a natural question is - whether the Lorentz-breaking field theory can be supersymmetric. A systematic methodology used to address this problem is based on the Kostelecky-Berger construction [3] involving the deformation of the supersymmetry algebra, which, in principle, can be applied to different kinds of superfield theories, formulated in different space-time dimensions, and allows for the arising of the CPT-even Lorentz-breaking terms on the component level. Other possible solution for this problem involve extra superfield(s) whose component(s) depend on the Lorentzbreaking parameters [4] (which can allow for the arising of the CPT-odd Lorentz-breaking terms on the component level), or a straightforward addition of Lorentz-breaking, superfield-dependent terms like $k^{a b} \partial_{a} \Phi \partial_{b} \bar{\Phi}$ (where $\Phi, \bar{\Phi}$ are the superfields, and the $k^{a b}$ is a constant tensor). To the best of our knowledge, however, the last method has not been systematically used yet, and it is clear that it involves higher derivatives.

In this paper we develop a method based on the Kostelecky-Berger (KB) construction, to introduce a Lorentz-breaking deformation of the supersymmetry (SUSY) algebra for supergauge field theories. Earlier, this method was successfully applied to supersymmetric scalar field theories [5]. There it was shown that the application of the KB construction allows to generate aether-like terms [6], in the action of the theories at the component level, while the effective action can be calculated on the basis of the superfield approach, in a way as simple as in the usual, Lorentzinvariant case. We develop this methodology, both in three- and in four-dimensional cases, and one of the key results of our consideration consists in a natural arising of a new form of gauge symmetry, involving the Lorentz-breaking parameter, for the vector component of the superfield.

The paper is organized as follows. In Sec. III we discuss the generalization of the threedimensional aether superspace to gauge theories, including some perturbative calculations on threedimensional supersymmetric quantum electrodynamics and Chern-Simons-matter model. In Sec. III we deal with the four-dimensional case, and we apply the aether superspace methodology in the computation of the effective potential to the supersymmetric quantum electrodynamics. In Sec. IV we discuss the possibility of equivalence between our modification of the supersymmetry generators and some coordinate transformations. Finally, in Sec. $\mathrm{V}$ we present our final remarks. 


\section{THREE-DIMENSIONAL AETHER SUPERSPACE}

Just as we have discussed in our previous paper [5], the extension of the usual superspace to a three-dimensional deformed superspace is stated through the deformed SUSY generators

$$
\begin{aligned}
Q_{\alpha} & =i\left[\partial_{\alpha}-i \theta^{\beta} \gamma_{\beta \alpha}^{m}\left(\partial_{m}+k_{m n} \partial^{n}\right)\right] \\
& =i\left[\partial_{\alpha}-i \theta^{\beta} \gamma_{\beta \alpha}^{m} \nabla_{m}\right]
\end{aligned}
$$

satisfying the anti-commutation relation

$$
\left\{Q_{\alpha}, Q_{\alpha}\right\}=2 i \gamma_{\alpha \beta}^{m} \nabla_{m}
$$

where $\partial_{\alpha}$ is the derivative with respect to the Grassmannian coordinates $\theta^{\alpha}$ and $\nabla_{m}=\partial_{m}+k_{m n} \partial^{n}$, with $\partial_{m}$ the derivative with respect to $x^{m}$. Latin indices assume values of three-dimensional spacetime coordinates $(0,1,2)$ and $k_{m n}$ is a constant tensor which can be chosen to assume an aether-like form $k_{m n}=\alpha u_{m} u_{n}$, with $\alpha$ a small parameter (cf. [6]) and $u^{m}$ being a constant vector with $u^{m} u_{m}$ equal either to $1,-1$ or 0 . In general, we use the conventions and notations as well as normalization factors as in Ref. 7], but the symbols for the (supergauge covariant) derivatives appearing in the present paper are slightly different.

It is important to remark that the new supercovariant derivative which anti-commute with $Q_{\alpha}$ is given by

$$
D_{\alpha}=\partial_{\alpha}+i \theta^{\beta} \gamma_{\beta \alpha}^{m} \nabla_{m}
$$

where the operator $\nabla_{m}$ commutes with $D_{\alpha}$, as well as with the SUSY generators.

For superscalar field theories constructed in this deformed superspace, we can define an action such as

$$
S=-\frac{1}{2} \int d^{5} z\left[\left(\overline{D^{\alpha} \Phi}\right)\left(D_{\alpha} \Phi\right)-f(\bar{\Phi} \Phi)\right],
$$

where $f(\bar{\Phi} \Phi)$ is some function of the bilinear $\bar{\Phi} \Phi$, which is invariant under $U(1)$ global transformations $\left(\Phi^{\prime}=\mathrm{e}^{i K} \Phi\right)$. Our aim in this paper is to extend such action to theories that are invariant under local (gauge) transformations $\left(\Phi^{\prime}=\mathrm{e}^{i K(x, \theta)} \Phi\right.$, with $K(x, \theta)$ being a real scalar superfield).

To do this, let us introduce a supergauge covariant derivative $\mathcal{D}_{\alpha}=\left(D_{\alpha}-i \Gamma_{\alpha}\right)$ such tht $\mathcal{D}_{\alpha} \Phi$ transforms covariantly under $U(1)$ gauge transformations $\left(\mathcal{D}_{\alpha}^{\prime} \Phi^{\prime}=\mathrm{e}^{i K(x, \theta)} \mathcal{D}_{\alpha} \Phi\right)$, allowing us to write a gauge invariant action

$$
\begin{aligned}
S & =-\frac{1}{2} \int d^{5} z\left[\left(\overline{\mathcal{D}^{\alpha}} \Phi\right)\left(\mathcal{D}_{\alpha} \Phi\right)-f(\bar{\Phi} \Phi)\right] \\
& =-\frac{1}{2} \int d^{5} z\left[\left(\overline{D^{\alpha} \Phi}\right)\left(D_{\alpha} \Phi\right)-i \overline{D^{\alpha} \Phi} \Gamma_{\alpha} \Phi+i \Gamma^{\alpha} \bar{\Phi} D_{\alpha} \Phi+\Gamma^{\alpha} \Gamma_{\alpha} \bar{\Phi} \Phi-f(\bar{\Phi} \Phi)\right],
\end{aligned}
$$


where the spinor gauge connection transforms as $\Gamma_{\alpha}^{\prime}=\Gamma_{\alpha}+D_{\alpha} K$. Note that the gauge transformations themselves are defined as deformed ones.

The components of the spinor superfield connection can be defined as

$$
\begin{gathered}
\chi_{\alpha}=\left.\Gamma_{\alpha}\right|_{\theta=0}, \quad B=\left.\frac{1}{2} D^{\alpha} \Gamma_{\alpha}\right|_{\theta=0}, \\
V_{\alpha \beta}=-\left.\frac{i}{2} D_{(\alpha} \Gamma_{\beta)}\right|_{\theta=0}, \quad \lambda_{\alpha}=\left.\frac{1}{2} D^{\beta} D_{\alpha} \Gamma_{\beta}\right|_{\theta=0},
\end{gathered}
$$

where $V_{\alpha \beta}=\left(\gamma^{m}\right)_{\alpha \beta} A_{m}$. In analogy with the usual three-dimensional superspace, the components of scalar superfield are conveniently defined as

$$
\varphi=\left.\Phi\right|_{\theta=0}, \quad \psi=\left.D^{\alpha} \Phi\right|_{\theta=0}, \quad F=\left.D^{2} \Phi\right|_{\theta=0},
$$

with similar definitions for the components of $\bar{\Phi}$.

Therefore, in terms of the components of the superfields, the action Eq.(15) can be cast as

$$
\begin{aligned}
S= & \int d^{3} x\left\{\bar{F} F+\bar{\psi}^{\alpha}\left(\gamma^{m}\right)_{\alpha}^{\beta}\left[i \nabla_{m}-A_{m}\right] \psi_{\beta}+\left(i \bar{\psi}^{\alpha} \lambda_{\alpha} \varphi+h . c .\right)\right. \\
& +\left(\nabla^{m}-i A^{m}\right) \bar{\varphi}\left(\nabla_{m}+i A_{m}\right) \varphi+\frac{1}{2} f^{\prime}(\bar{\varphi} \varphi)\left[\bar{F} \varphi+\bar{\varphi} F+2 \bar{\psi}^{\beta} \psi_{\beta}\right] \\
& \left.+\frac{1}{2} f^{\prime \prime}(\bar{\varphi} \varphi)\left[2 \bar{\varphi} \varphi \bar{\psi}^{\beta} \psi_{\beta}+\varphi^{2} \bar{\psi}^{\beta} \bar{\psi}_{\beta}+\bar{\varphi}^{2} \psi^{\beta} \psi_{\beta}\right]\right\},
\end{aligned}
$$

where $f^{\prime}(\bar{\varphi} \varphi)=\left.\frac{\partial f(\bar{\Phi} \Phi)}{\partial(\bar{\Phi} \Phi)}\right|_{\bar{\Phi} \Phi=\bar{\varphi} \varphi}$.

Gauge covariant superfield strength can be defined just as in the usual case, $W_{\alpha}=\frac{1}{2} D^{\beta} D_{\alpha} \Gamma_{\beta}$. A SUSY Maxwell Lorentz-breaking action can be constructed as

$$
S=\int d^{5} z \frac{1}{2} W^{\alpha} W_{\alpha}=\int d^{3} x\left[\lambda^{\alpha} i\left(\gamma^{m}\right)_{\alpha}^{\beta} \nabla_{m} \lambda_{\beta}-\frac{1}{2} f^{\alpha \beta} f_{\alpha \beta}\right],
$$

where $\left.\lambda_{\alpha} \equiv W_{\alpha}\right|_{\theta=0}$ and $f_{\alpha \beta}=\left.D_{\alpha} W_{\beta}\right|_{\theta=0}=\left.D_{\beta} W_{\alpha}\right|_{\theta=0}$. In terms of the gauge field $A_{m}, f_{\alpha \beta}$ can be written as $f_{\alpha \beta}=\frac{1}{2} \epsilon^{m n}{ }_{r}\left(\gamma^{r}\right)_{\alpha \beta} \nabla_{m} A_{n}$. Therefore the physical content of the SUSY Maxwell-like action is given by

$$
S=\int d^{3} x\left[\lambda^{\alpha} i\left(\gamma^{m}\right)_{\alpha}{ }^{\beta} \nabla_{m} \lambda_{\beta}-\frac{1}{4}\left(\nabla_{m} A_{n}-\nabla_{n} A_{m}\right)^{2}\right],
$$

where $\nabla_{m}=\partial_{m}+k_{m n} \partial^{n}$.

\section{A. Three-dimensional quantum electrodynamics in aether superspace}

As a first example of the power of the superspace techniques even in Lorentz breaking scenarios, let us evaluate the one-loop correction to the self-energy of the gauge superfield in the 
Super Quantum Electrodynamics in three-dimensions. To do this it is necessary to compute the superpropagators of the model. Considering the theory defined by the action Eq.(5)+9) plus the gauge-fixing and the corresponding Fadeev-Popov term, and proceeding as usual, we can write the following propagators in the aether superspace

$$
\begin{aligned}
\left\langle\Phi\left(p, \theta_{1}\right) \Phi\left(-p, \theta_{2}\right)\right\rangle & =\frac{\left(D^{2}-m\right)}{\tilde{p}^{2}+m^{2}} \delta^{2}\left(\theta_{1}-\theta_{2}\right), \\
\left\langle\Gamma_{\alpha}\left(p, \theta_{1}\right) \Gamma_{\beta}\left(-p, \theta_{2}\right)\right\rangle & =\frac{1}{\tilde{p}^{2}}\left[\frac{(1+\xi)}{2} C_{\beta \alpha}-\frac{(1-\xi)}{2} \frac{\left(\gamma^{m}\right)_{\beta \alpha} \tilde{p}_{m} D^{2}}{\tilde{p}^{2}}\right] \delta^{2}\left(\theta_{1}-\theta_{2}\right),
\end{aligned}
$$

where $\tilde{p}_{m}=p_{m}+k_{m n} p^{n}, \tilde{p}^{2}=p^{2}+2 k_{m n} p^{m} p^{n}+k^{m n} k_{m l} p_{n} p^{l}, D^{2}=\partial^{2}-\theta^{\beta}\left(\gamma^{m}\right)_{\beta \alpha} \tilde{p}_{m} \partial^{\alpha}+\theta^{2} \tilde{p}^{2}$. As we commented in our previous work [5], this dispersion relation has a structure common for the propagators in the CPT-even Lorentz-breaking theories (see e.g. [8]).

We are able to compute the radiative corrections to the Super Quantum Electrodynamics Lorentz-breaking theory, Eq.(9). and choosing $f(\bar{\Phi} \Phi)=M \bar{\Phi} \Phi$ (i.e., a mass term to the scalar superfield), the diagrams which contribute to the effective action is depicted in Fig,1. The corresponding expression can be cast as

$$
S_{2 l}=\int \frac{d^{3} p}{(2 \pi)^{3}} d^{2} \theta\left[W^{\alpha} W_{\alpha}-M \Gamma^{\alpha} W_{\alpha}\right] \int \frac{d^{3} q}{(2 \pi)^{3}} \frac{1}{\left(\tilde{q}^{2}+M^{2}\right)\left[(\tilde{q}-\tilde{p})^{2}+M^{2}\right]} .
$$

This last integral can be evaluated by changing the variable of integration $q$ to $\tilde{q}$. In the case of $p^{2} \approx 0$ we can write $\int d^{3} q=\Delta \int d^{3} \tilde{q}$, where $\Delta=\operatorname{det}\left(\frac{\partial q^{m}}{\partial \tilde{q}^{n}}\right)=\operatorname{det}^{-1}\left(\delta_{n}^{m}+k_{n}^{m}\right)$ is the Jacobian of the changing of variables. For $k_{m n}=\alpha u_{m} u_{n}$ with a small $\alpha, \Delta \approx\left(1-\alpha u^{2}\right)$. So, the final result is

$$
S_{2 l}=\frac{\Delta}{8 \pi|M|} \int \frac{d^{3} p}{(2 \pi)^{3}} d^{2} \theta\left[W^{\alpha} W_{\alpha}-M \Gamma^{\alpha} W_{\alpha}\right] .
$$

We can observe that the one-loop quantum correction is finite. This model is known to be finite to all loop orders in perturbation theory in the usual superspace [9, 10], and it is natural to expect that this issue persists in the aether superspace, since the power counting of the model is not affected by the presence of the Lorentz breaking terms introduced through the aether superspace. We also observe, the generation of a Super-Chern-Simons Lorentz-breaking term, which corresponding bosonic local part has the form

$$
\int d^{3} x M \Delta \epsilon^{l m n} A_{l} \nabla_{m} A_{n}=\int d^{3} x M \Delta \epsilon^{l m n}\left[A_{l} \partial_{m} A_{n}+k_{m s} A_{l} \partial^{s} A_{n}\right] .
$$

We note that the Chern-Simons action, instead of the usual gauge transformations, is invariant under the new ones $\delta A_{n}=\nabla_{n} \xi$, with $\xi$ being a parameter of the gauge transformation.

Let us now, discuss the Maxwell action. It is easy to see that, after doing the Fourier transformation and reducing to the component fields, the Maxwell-like contribution from (13) looks 
like

$$
S_{M}=-\frac{1}{4} \frac{\Delta}{8 \pi|M|} \int d^{3} x \tilde{F}_{m n} \tilde{F}^{m n}
$$

where

$$
\tilde{F}_{m n}=\nabla_{m} A_{n}-\nabla_{n} A_{m}
$$

is a new stress tensor. We note that the derivatives $\nabla_{m}$ emerge from the supercovariant spinor derivatives. So, as can be seen, a new action for the vector field $A_{m}$ is generated, that is (as the Chern-Simons one) invariant under the new gauge transformations $\delta A_{n}=\nabla_{n} \xi$. We note that the action (15) essentially differs from the usual aether-like action for the gauge field [6] which is invariant under the usual gauge transformations $\delta A_{n}=\partial_{n} \xi$, and cannot be reduced to it.

\section{B. Chern-Simons-matter model in aether Superspace}

Quantum field theories defined in a three-dimensional space-time are widely discussed in the literature because they offer a very rich structure, working as excellent theoretical laboratories as well as they can be applied to some almost planar condensed matter systems, such as quantum Hall effect [11]. More recently, supersymmetric gauge field theories in three dimensions could be related to M2-branes [12 14]. Of special interest is the computation of the effective superpotential of the Supersymmetric Chern-Simons-matter model [15 17, 19 21], that can be used to evaluate the possibility of spontaneous (super)symmetry breaking via Coleman-Weinberg mechanism [22]. The presence of Lorentz symmetry violating terms in the Lagrangian could be a source of spontaneous SUSY breaking [23] .

Let us start by defining the classical action of the model

$$
S=\int d^{5} z\left\{\Gamma^{\alpha} W_{\alpha}-\frac{1}{2} \overline{\mathcal{D}^{\alpha} \Phi} \mathcal{D}_{\alpha} \Phi+\lambda(\bar{\Phi} \Phi)^{2}\right\},
$$

where $W^{\alpha}=(1 / 2) D^{\beta} D^{\alpha} \Gamma_{\beta}$ is the gauge superfield strength as defined before and $\mathcal{D}^{\alpha}=\left(D^{\alpha}-i e \Gamma^{\alpha}\right)$ is the supercovariant derivative.

The action Eq.(17) possesses manifest $\mathcal{N}=1$ SUSY, and it can be lifted to $\mathcal{N}=2$ by the elimination of the fermion-number violating terms [24], from which we identify the coupling constants as $\lambda=-e^{2} / 8$. In the usual superspace, SCSM is superconformal invariant at classical level, but in the aether superspace the presence of the constant vector $k_{m n}$ explicitly breaks this invariance; even so, we should expect that an analog (or extended) symmetry could emerge from the action Eq.(17), but we will not extend such analysis in this paper. 
The presence of a mass term like $\int d^{5} z M \bar{\Phi} \Phi$, with a wrong sign, in the action Eq.(17) would generate a spontaneously (gauge) symmetry broken phase at classical level [25], but here we are interested in spontaneous symmetry breaking induced by radiative corrections (Coleman-Weinberg mechanism), and so we will keep the model massless at this level. To this end, let us shift the superfields $\bar{\Phi}$ and $\Phi$ by the classical background superfield $\varphi$ as

$$
\begin{aligned}
& \bar{\Phi}=\frac{1}{\sqrt{2}}\left(\varphi+\Phi_{1}-i \Phi_{2}\right) \\
& \Phi=\frac{1}{\sqrt{2}}\left(\varphi+\Phi_{1}+i \Phi_{2}\right)
\end{aligned}
$$

where $\varphi=\varphi_{1}-\theta^{2} \varphi_{2}$, with $\varphi_{1}$ and $\varphi_{2}$ being real constant fields.

Assuming the vanishing of the vacuum expectation values (VEV) of the quantum superfields, i.e., $\left\langle\Phi_{1}\right\rangle=\left\langle\Phi_{2}\right\rangle=0$ at any order of perturbation theory, the gauge invariant action Eq.(17) results in,

$$
\begin{aligned}
S & =\int d^{5} z\left\{\Gamma^{\alpha} W_{\alpha}-\frac{e^{2} \varphi^{2}}{4} \Gamma^{\alpha} \Gamma_{\alpha}-\frac{e \varphi}{2} D^{\alpha} \Gamma_{\alpha} \Phi_{2}+\frac{1}{2} \Phi_{1}\left(D^{2}+3 \lambda \varphi^{2}\right) \Phi_{1}+\frac{1}{2} \Phi_{2}\left(D^{2}+\lambda \varphi^{2}\right) \Phi_{2}\right. \\
& +\frac{1}{2} \varphi D^{2} \varphi+\frac{\lambda}{4} \varphi^{4}+\frac{e}{2} D^{\alpha} \Phi_{2} \Gamma_{\alpha} \Phi_{1}-\frac{e}{2} D^{\alpha} \Phi_{1} \Gamma_{\alpha} \Phi_{2}-\frac{e^{2}}{2}\left(\Phi_{1}^{2}+\Phi_{2}^{2}\right) \Gamma^{2}-e^{2} \varphi \Phi_{1} \Gamma^{2} \\
& +\frac{\lambda}{4}\left(\Phi_{1}^{4}+\Phi_{2}^{4}\right)+\frac{\lambda}{2} \Phi_{1}^{2} \Phi_{2}^{2}+\lambda \varphi \Phi_{1}\left(\Phi_{1}^{2}+\Phi_{2}^{2}\right)-e D^{\alpha} \varphi \Phi_{2} \Gamma_{\alpha}+\left(\lambda \varphi^{3}+D^{2} \varphi\right) \Phi_{1} \\
& \left.+\frac{1}{2 \alpha}\left(D^{\alpha} \Gamma_{\alpha}+\alpha \frac{e \varphi}{2} \Phi_{2}\right)^{2}+\bar{c} D^{2} c+\frac{\alpha}{4} e^{2} \varphi^{2} \bar{c} c+\frac{\alpha}{4} e^{2} \varphi \bar{c} \Phi_{1} c\right\} .
\end{aligned}
$$

In the last line we added a gauge fixing and the corresponding Faddeev-Popov terms. We have used an $R_{\xi}$ gauge condition to eliminate the mixing between $\Gamma$ and $\Phi_{2}$ superfields, but this procedure is not enough to completely eliminate this mixing. Even so, the remaining term, $-e D^{\alpha} \varphi \Phi_{2} \Gamma_{\alpha}$, can be disregarded in the Kählerian approximation of the effective superpotential, because it contains a supercovariant derivative applied to the background superfield $\varphi$.

The knowledge of the Kählerian effective superpotential is enough to determine the possibility of spontaneous SUSY and gauge symmetry breaking [18, 19]. We will evaluate it at two-loop order, where such effects are expected to show up [19, 26, 27].

The Feynman rules derived from Eq.(19) are given, in the Kählerian approximation (that is, by 
preserving the dependence in $\varphi$ and dropping the dependences on $D_{\alpha} \varphi$ and $D^{2} \varphi$ ), by

$$
\begin{aligned}
\left\langle T \Phi_{1}(k, \theta) \Phi_{1}\left(-k, \theta^{\prime}\right)\right\rangle & =-i \frac{D^{2}-M_{\Phi_{1}}}{k^{2}+M_{\Phi_{1}}^{2}} \delta^{(2)}\left(\theta-\theta^{\prime}\right), \\
\left\langle T \Phi_{2}(k, \theta) \Phi_{2}\left(-k, \theta^{\prime}\right)\right\rangle & =-i \frac{D^{2}-M_{\Phi_{2}}}{k^{2}+M_{\Phi_{2}}^{2}} \delta^{(2)}\left(\theta-\theta^{\prime}\right) \\
\left\langle T \Gamma_{\alpha}(k, \theta) \Gamma_{\beta}\left(-k, \theta^{\prime}\right)\right\rangle & =\frac{i}{4}\left[\frac{\left(D^{2}+M_{A}\right) D^{2} D_{\beta} D_{\alpha}}{k^{2}\left(k^{2}+M_{\Gamma}^{2}\right)}\right. \\
& \left.+\alpha \frac{\left(D^{2}-\alpha M_{\Gamma}\right) D^{2} D_{\alpha} D_{\beta}}{k^{2}\left(k^{2}+\alpha^{2} M_{\Gamma}^{2}\right)}\right] \delta^{(2)}\left(\theta-\theta^{\prime}\right) .
\end{aligned}
$$

For simplicity, let us choose the SUSY Landau gauge $\alpha=0$ (we have to remark that the effective superpotential is a gauge-dependent quantity [28]). With this choice, the ghost superfields are decoupled from the model, and we can identify the poles of the propagators of the interacting superfields as

$$
M_{\Phi_{1}}=3 \lambda \varphi^{2}, \quad M_{\Phi_{2}}=\lambda \varphi^{2}, \quad M_{\Gamma}=\frac{e^{2} \varphi^{2}}{4} .
$$

Proceeding as described in [29], considering the two-loop corrections depicted in Fig 2, and performing the integrals using the regularization by dimensional reduction [30], the two-loop Kählerian effective superpotential can be cast as

$$
K(\varphi)=-\frac{b_{2}}{4} \varphi^{4}\left(\frac{b_{1}}{b_{2}}-\frac{1}{2} \Delta^{2}+\Delta^{2} \ln \frac{\varphi^{2}}{\mu}\right)-\frac{B}{4} \varphi^{4} .
$$

where $B$ is a counterterm, $\mu$ is a mass scale introduced by the regularization, $b_{1}$ is a function of the coupling constants, of $\Delta$ and of $1 / \epsilon \equiv \frac{1}{3-D}$ ( $D$ is the dimension of the space-time). The quantity $b_{2}$ is explicitly given by

$$
b_{2}=-\left(116 e^{6}+543 e^{4} \lambda+432 e^{2} \lambda^{2}-71552 \lambda^{3}\right) /\left(12288 \pi^{2}\right) .
$$

The counterterm $B$ is fixed through the following renormalization condition

$$
\left.\frac{\lambda}{4} \equiv \frac{1}{4 !} \frac{\partial^{4} K(\varphi)}{\partial \varphi^{4}}\right|_{\varphi=v}
$$

where $v$ is the renormalization point. By substituting $B$ in Eq. (22), the Kählerian effective superpotential results in

$$
K(\varphi)=-\frac{b_{2} \Delta^{2}}{4} \varphi^{4} \ln \left[\frac{\varphi^{2}}{v^{2}} \exp \left(-\frac{\lambda}{b_{2} \Delta^{2}}-\frac{25}{6}\right)\right] .
$$

Now we are able to study the spontaneous generation of mass to the physical superfields induced by the radiative corrections. First, let us impose the condition to extremize the Kählerian effective superpotential. It reads

$$
\frac{\partial K(\varphi)}{\partial \varphi}=\frac{\varphi^{3}}{3}\left[3 \lambda+3 b_{2} \Delta^{2}\left(\frac{11}{3}-\ln \frac{\varphi^{2}}{v^{2}}\right)\right]=0 .
$$


The nontrivial solutions are given by

$$
\varphi= \pm v \exp \left(\frac{11}{6}+\frac{\lambda}{2 b_{2} \Delta^{2}}\right)
$$

Since we have computed the effective superpotential for constant configurations of the background superfields, we expect that our approximation is valid for small fluctuations around the mass scale $v$, used as renormalization point. This expectative constraints the exponential of the above equation to be approximately 1 . Therefore, we want that the coupling constants satisfy $\frac{11}{6}+\frac{\lambda}{2 b_{2} \Delta^{2}} \approx 0$, what results in the following condition

$$
\lambda \approx \frac{11}{3} b_{2} \Delta^{2} \approx\left[-\left(4 \times 10^{-3}\right) e^{6}-\left(16 \times 10^{-3}\right) e^{4} \lambda-\left(13 \times 10^{-3}\right) e^{2} \lambda^{2}+2 \lambda^{3}\right] \Delta^{2} .
$$

For the Coleman-Weinberg mechanism, this last equation is directly related to the compatibility of the effective superpotential calculations with the assumptions of perturbation theory. We can see from Eq. (28) that $\lambda$ should be of order of $\left(4 \times 10^{-3}\right) e^{6} \Delta^{2}+\mathcal{O}\left(e^{10}\right)$, so that, for small $e$ we are in the regime of validity of the perturbative expansion.

The second derivative of the Kählerian effective superpotential with respect to the background field $\varphi$ evaluated in the minimum of the superpotential, i.e. $\varphi \simeq \pm v$, is interpreted as the mass of the matter (background) superfield $\varphi$. If positive, this condition guarantees that Eq.(27) is a minimum of $K(\varphi)$. In fact, using Eqs. (28) and (28), we obtain

$$
M_{\varphi}=\left.\frac{d^{2} K(\varphi)}{d \varphi^{2}}\right|_{\varphi=v} \approx\left(2 \times 10^{-3}\right) e^{6} v^{2} \Delta^{2}
$$

and the mass of the gauge superfield induced by the radiative corrections is given by

$$
\frac{M_{\Gamma}}{M_{\varphi}} \sim \frac{e^{2}}{12 \lambda} \sim-21 \frac{e^{-4}}{\Delta^{2}}
$$

where we can notice that the mass of the gauge superfield is much larger than the mass of matter superfield since, for a small violation of the Lorentz symmetry, $\Delta$ should be approximately 1 .

One interesting remark is that all information of the presence of Lorentz violating terms in the original action is manifested in the presence of the $\Delta$ factor in the effective superpotential, and consequently in the induced masses. Since the Kählerian effective superpotential has a consistent minimum, we can affirm that SUSY can not be spontaneously broken via Coleman-Weinberg mechanism. Probably, to search for SUSY breaking induced by the Lorentz violating terms we should compute the whole effective action, using some more sophisticated technology. 


\section{FOUR-DIMENSIONAL AETHER SUPERSPACE}

Now, let us consider gauge theories within the four-dimensional aether superspace. In this case, the spinor supercovariant derivatives look like

$$
\begin{aligned}
& D_{\alpha}=\partial_{\alpha}+i \bar{\theta}^{\dot{\beta}} \sigma_{\dot{\beta} \alpha}^{m} \nabla_{m} \\
& \bar{D}_{\dot{\alpha}}=\partial_{\dot{\alpha}}+i \theta^{\beta} \bar{\sigma}_{\beta \dot{\alpha}}^{m} \nabla_{m},
\end{aligned}
$$

where again $\nabla_{m}=\partial_{m}+k_{m n} \partial^{n}$. It is clear that these spinor derivatives satisfy the usual properties

$$
D_{\alpha} D_{\beta} D_{\gamma}=0 ; \quad \bar{D}_{\dot{\alpha}} \bar{D}_{\dot{\beta}} \bar{D}_{\dot{\gamma}}=0
$$

Now, let us define the following Abelian gauge theory

$$
S=\int d^{6} z W^{\alpha} W_{\alpha}
$$

where

$$
W_{\alpha}=\frac{1}{8} \bar{D}^{2}\left(e^{-v} D_{\alpha} e^{v}\right)=\frac{1}{8} \bar{D}^{2} D_{\alpha} v
$$

In principle, the non-Abelian generalization of this theory can be constructed along the same lines.

This action can be rewritten as

$$
S_{W}=-\frac{1}{16} \int d^{8} z v D^{\alpha} \bar{D}^{2} D_{\alpha} v
$$

As can be seen, its form does not differ from the usual action of gauge theories (see f.e. [7]); the only difference, from the usual case, consists in the replacement of the common spinor supercovariant derivative by a new one given by (31). It is clear that this action is invariant under the gauge transformations $\delta v=\Lambda+\bar{\Lambda}$, where $\Lambda$ is a chiral superfield, and $\bar{\Lambda}$ is an antichiral one.

Following the general principles, we suggest that the component expansion of the real scalar superfield $v$ is the same as in the usual case, i.e. it depends on the relevant vector (gauge) field $A_{m}$ as

$$
v=-\frac{i}{2}\left(\bar{\theta} \sigma^{m} \theta\right) A_{m}(x)+\ldots
$$

By reducing the action (35) to the component fields, its bosonic part can be shown to have the form (15) up to the numerical factor, with the only difference that the integral is now performed over the four-dimensional space-time. 
Then, we must add the following gauge-fixing action

$$
S_{g f}=\frac{1}{16 \alpha} \int d^{8} z v D^{2} \bar{D}^{2} v
$$

where $\alpha$ is the gauge-fixing parameter.

The corresponding propagator looks like

$$
<v\left(z_{1}\right) v\left(z_{2}\right)>=-\frac{1}{\tilde{\square}}\left(-\frac{D^{\alpha} \bar{D}^{2} D_{\alpha}}{8 \tilde{\square}}+\alpha \frac{\left\{\bar{D}^{2}, D^{2}\right\}}{16 \tilde{\square}}\right) \delta^{8}\left(z_{1}-z_{2}\right),
$$

involving the new projection operators

$$
\Pi_{0}=\frac{\left\{\bar{D}^{2}, D^{2}\right\}}{16 \tilde{\square}}, \quad \Pi_{1 / 2}=-\frac{D^{\alpha} \bar{D}^{2} D_{\alpha}}{8 \tilde{\square}} .
$$

Then, we couple the gauge field to the chiral matter filed $\phi$, by introducing the following action

$$
S_{\Phi}=\int d^{8} z \bar{\phi} e^{g v} \phi
$$

The propagators of the chiral field look like (cf. [5]):

$$
<\phi\left(z_{1}\right) \bar{\phi}\left(z_{2}\right)>=\frac{\bar{D}^{2} D^{2}}{16 \tilde{\square}} \delta^{8}\left(z_{1}-z_{2}\right) \quad<\bar{\phi}\left(z_{1}\right) \phi\left(z_{2}\right)>=\frac{D^{2} \bar{D}^{2}}{16 \tilde{\square}} \delta^{8}\left(z_{1}-z_{2}\right) .
$$

To calculate the one-loop Kählerian effective potential, we can use the well-developed methodology of calculating the superfield effective potential elaborated in 31 33]. As usual, one can begin with constructing the one-loop Feynman diagrams contributing to the superfield effective potential. The structure of the supergraphs does not essentially differ from the usual case [31]. The first set depicted at Fig. 3 involves only gauge propagators. Their sum is given by

$$
K_{a}^{(1)}=\left.\int d^{8} z_{1} \sum_{n=1}^{\infty} \frac{(-1)^{n}}{2 n}\left(g^{2} \Phi \bar{\Phi} \frac{1}{\square}\left(\Pi_{1 / 2}+\alpha \Pi_{0}\right)\right)^{n} \delta_{12}\right|_{\theta_{1}=\theta_{2}},
$$

where $\frac{1}{n}$ is a symmetry factor.

Proceeding just as in [31], we find that

$$
K_{a}^{(1)}=\int d^{8} z \int \frac{d^{4} p}{(2 \pi)^{4}} \frac{1}{\tilde{p}^{2}}\left[\ln \left(1+\frac{g^{2} \Phi \bar{\Phi}}{\tilde{p}^{2}}\right)-\ln \left(1+\frac{\alpha g^{2} \Phi \bar{\Phi}}{\tilde{p}^{2}}\right)\right]
$$

where $\tilde{p}^{2}=\left(p_{m}+k_{m n} p^{n}\right)^{2}$ is a Fourier transform for $\tilde{\square}$. Notice that at $\alpha=0$ (Landau gauge), the second term in (42) vanishes. Using the notations adopted in [34], one can introduce a "dressed" propagator involving a sum over quartic vertices (see Fig. 4):

$$
<v v>_{D}=-\left(\frac{1}{\tilde{\square}+g^{2} \Phi \bar{\Phi}} \Pi_{1 / 2}+\frac{\alpha}{\tilde{\square}+\alpha g^{2} \Phi \bar{\Phi}} \Pi_{0}\right) \delta^{8}\left(z_{1}-z_{2}\right) .
$$


The triple vertices will enter the Feynman diagrams only through the links depicted at Fig. 5, and the contribution from this sector is given by the sum of the supergraphs depicted at Fig. 6 . It is equal to

$$
K_{b}^{(1)}=\int d^{8} z \int \frac{d^{4} p}{(2 \pi)^{4}} \frac{1}{\tilde{p}^{2}}\left[\ln \left(1+\frac{\alpha g^{2} \Phi \bar{\Phi}}{\tilde{p}^{2}}\right)\right]
$$

The total result which is the sum of $K_{a}^{(1)}$ and $K_{b}^{(1)}$, is gauge invariant and equal to

$$
K^{(1)}=\int d^{8} z \int \frac{d^{4} p}{(2 \pi)^{4}} \frac{1}{\tilde{p}^{2}} \ln \left(1+\frac{g^{2} \Phi \bar{\Phi}}{\tilde{p}^{2}}\right) .
$$

To calculate these integrals, we can change the variables as in [5]. After integration and subtracting the divergences we arrive at

$$
K^{(1)}=-\frac{1}{32 \pi^{2}} \Delta g^{2} \Phi \bar{\Phi} \ln \frac{g^{2} \Phi \bar{\Phi}}{\mu^{2}}
$$

where $\Delta$ is again a Jacobian of the change of variables $k^{m} \rightarrow \tilde{k}^{m}$. We see that the result only differs from the usual case [32], by the multiplicative factor $\Delta$.

\section{LORENTZ-BREAKING MODIFICATION OF THE SUPERSYMMETRY GENERA- TORS AND COORDINATE TRANSFORMATIONS}

To close the paper, let us discuss the possible impacts of the Lorentz-breaking modification of the supersymmetry generators for the generic quantum contributions to an effective action of arbitrary superfield theory. It follows from the the definitions of modified supersymmetry generators and covariant derivatives (1131) that the methodology of Lorentz symmetry breaking adopted by us implies the change of all momenta, that emerge through the D-algebra transformations, by the rule $p_{m} \rightarrow p_{m}+k_{m n} p^{n}$, or, in the coordinate space, $\partial_{m} \rightarrow \partial_{m}+k_{m n} \partial^{n}$ (which corresponds to the linear coordinate change $\left.x_{m} \rightarrow\left(\delta_{m}^{n}+k_{m}^{n}\right)^{-1} x_{n}\right)$. Therefore one can elaborate the following geometric interpretation of this Lorentz-breaking modification of SUSY algebra.

It was shown in our previous paper [5] that the one-loop contribution to two-point function in a $3 D$ self-coupled scalar superfield model looks like

$$
\Gamma_{2}^{(1)}=\frac{\Delta}{8 \pi|m|} \int d^{3} x d^{2} \theta \Phi\left(D^{2}-2 m\right) \Phi .
$$

By projecting this action to components, we arrive at

$$
\Gamma_{2}^{(1)}=\frac{\Delta}{8 \pi|m|} \int d^{3} x\left(-\eta^{m n} \nabla_{m} \phi \nabla_{n} \phi+\psi^{\alpha} i\left(\gamma^{m}\right)_{\alpha}^{\beta} \nabla_{m} \psi_{\beta}+F^{2}-2 m\left(\psi^{2}+\phi F\right)\right) .
$$


Let us perform, for this action, the analysis carried out in [35] It is clear that one can formally introduce the upper-index metric

$$
g^{a b}=\eta^{m n}\left(\delta_{m}^{a}+k_{m}^{a}\right)\left(\delta_{n}^{b}+k_{n}^{b}\right),
$$

with $g_{a b}$ introduced as usual to be the inverse of $g^{a b}$. The Jacobian $\Delta=\operatorname{det}^{-1}\left(\delta_{a}^{m}+k_{a}^{m}\right)$ can naturally be treated as a contribution to the integral measure, since $\Delta=\sqrt{\left|\operatorname{det} g_{a b}\right|}=\sqrt{|g|}$. One can also introduce the modified Dirac matrices $\tilde{\gamma}^{m}=\gamma^{m}+k_{n}^{m} \gamma^{n}$ which satisfy the modified anticommutation relation

$$
\left\{\tilde{\gamma}^{a}, \tilde{\gamma}^{b}\right\}=2 g^{a b},
$$

where $g^{a b}$ is given by (49). Therefore, the action (48) can be rewritten as

$$
\Gamma_{2}^{(1)}=\frac{1}{8 \pi|m|} \int d^{3} x \sqrt{|g|}\left(-g^{m n} \partial_{m} \phi \partial_{n} \phi+\psi^{\alpha} i\left(\tilde{\gamma}^{m}\right)_{\alpha}^{\beta} \partial_{m} \psi_{\beta}+F^{2}-2 m\left(\psi^{2}+\phi F\right)\right) .
$$

Therefore, we can say that, for the scalar superfield, our Lorentz-breaking modification of the supersymmetry generators is equivalent to introduction of a new metric, and, therefore, of a new geometry (this is an affine geometry since the new metric is related to the Minkowski one through a constant matrix). It is easy to check that an analogous situation occurs also in the four-dimensional chiral superfield theory, that is, in our extension of the Wess-Zumino model.

However, the situation differs for the contributions involving external gauge legs. We have shown above that in the $3 D$ gauge theory, the quantum correction is given by the expression (13). If we project it into components, the result in the purely gauge sector will be

$$
S_{M}=\frac{\Delta}{8 \pi|M|} \int d^{3} x\left(-\frac{1}{4} \eta^{m a} \eta^{n b} \tilde{F}_{m n} \tilde{F}_{a b}-M \epsilon^{a b c} A_{a} \nabla_{b} A_{c}\right) .
$$

Let us consider the Maxwell term, and, more precisely, one contribution to it, for example,

$$
S_{M 1}=\frac{\Delta}{8 \pi|M|} \int d^{3} x\left(-\frac{1}{4}\right) \eta^{m a} \eta^{n b} \nabla_{m} A_{n} \nabla_{a} A_{b} .
$$

Repeating identically the arguments above, we can rewrite this expression as

$$
S_{M 1}=\frac{1}{8 \pi|M|} \int d^{3} x \sqrt{|g|}\left(-\frac{1}{4}\right) g^{m a} \eta^{n b} \partial_{m} A_{n} \partial_{a} A_{b} .
$$

We see that while we succeeded in to replace the Minkowski metric to a new metric $g^{a b}$ in a sector involving only the space-time derivatives, there is no manner of to form a new "curved" metric $g^{a b}$ in a sector involving vector fields. This is related to the fact that within our methodology only the geometry (that is, coordinates, derivatives, metric and Dirac matrices) suffer transformations due to the introduction of the Lorentz-breaking parameters $k_{a b}$, but not the vector fields. Thus, there is no way to re-absorb the Lorentz breaking completely within a corresponding coordinate transformation in the gauge sector. This conclusion is similar to that one performed in [35]. 


\section{SUMMARY}

In this work we developed a gauge superfield method to construct Lorentz-breaking supersymmetric field theories based on the Kostelecky-Berger construction [3]. The methodology of superfields is a powerful tool to study, among others, perturbative aspects of supersymmetric theories. Even though SUSY and its algebra is closely related to Lorentz symmetry, we could extend the superfield formalism to include the Lorentz violating terms, therefore allowing us to use the most attractive properties of superspace formalism. The aether superspace is a natural way to deal with Lorentz violating supersymmetric models. In this context, we presented some applications of the aether superspace techniques in three and four-dimensional space-time, discussing perturbative aspects of supersymmetric quantum electrodynamics and Super-Chern-Simons-matter model. We showed that, from the methodological viewpoint, the calculations do not essentially differ from the usual Lorentz-invariant case. However, as we have noted, the new theory involving the Lorentz symmetry breaking can be reduced, though simple rules, to the usual Lorentz invariant theory, only if it is being considered in the purely scalar sector. If one deal with vector or spinor fields whose action involve metric contracted to fields, the redefinition of coordinates will not allow to redefine completely all the action, since it will imply in variations of the fields which are not suggested by the initial structure of our modification of the supersymmetry generators, cf. [35].

Acknowledgments. This work was partially supported by Conselho Nacional de Desenvolvimento Científico e Tecnológico $(\mathrm{CNPq})$, Fundação de Amparo à Pesquisa do Estado de São Paulo (FAPESP) and Fundação de Apoio à Pesquisa do Rio Grande do Norte (FAPERN). A. Yu. P. has been supported by the CNPq project 303438/2012-6.

[1] W. Bietenholz, Phys. Rep. 505, 145 (2011), arXiv: 0806.3713.

[2] V. A. Kostelecky, Phys. Rev. D69, 105009 (2004), hep-th/0312310.

[3] M. S. Berger, V. A. Kostelecky, Phys. Rev. D65, 091701 (2002), hep-th/0112243.

[4] H. Belich, J. L. Boldo, L. P. Colatto, J. A. Helayel-Neto, A. L. M. A. Nogueira, Phys.Rev. D68, 065030 (2003), hep-th/0304166.

[5] C. F. Farias, A. C. Lehum, J. R. Nascimento and A. Yu. Petrov, Phys. Rev. D 86, 065035 (2012) arXiv:1206.4508 [hep-th]].

[6] S. M. Carroll, H. Tam, Phys. Rev. D78, 044047 (2008), arXiv: 0802.0521; M. Gomes, J. R. Nascimento, 
A. Yu. Petrov, A. J. da Silva, Phys. Rev. D 81, 045018 (2010), arXiv: 0911.3548.

[7] S. J. Gates, M. T. Grisaru, M. Rocek, W. Siegel, Superspace or One Thousand and One Lessons in Supersymmetry, Benjamin/Cummings, 1983.

[8] R. Casana, M. M. Ferreira, A. R. Gomes, P. R. D. Pinheiro, Phys. Rev. D80, 125040 (2009), arXiv: 0905.0544 [hep-th], R. Casana, M. M. Ferreira, A. R. Gomes, F. dos Santos, Phys. Rev. D82, 125006 (2010), arXiv: 1010.2776 [hep-th]; R. Casana, M. M. Ferreira, R. P. M. Moreira, Phys. Rev. D84, 125014 (2011), arXiv: 1108.6193 [hep-th].

[9] L. P. Colatto, M. A. de Andrade, O. M. Del Cima, D. H. T. Franco, J. A. Helayel-Neto, O. Piguet, J. Phys. G24, 1301 (1998), hep-th/9712100.

[10] A. C. Lehum, A. F. Ferrari, M. Gomes, A. Yu. .Petrov and A. J. da Silva, Phys. Rev. D 77, 065005 (2008), arXiv: 0709.3501.

[11] "The Quantum Hall Effect", Graduate Texts in Contemporary Physics, R.E. Prange and S.M. Girvin eds, Springer-Verlag, Berlin, 1990.

[12] J. Bagger and N. Lambert, Phys. Rev. D 75, 045020 (2007).

[13] J. A. Bagger and N. Lambert, Phys. Rev. D 77, 065008 (2008).

[14] A. Gustavsson, Nucl. Phys. B 811, 66 (2009).

[15] M. Gomes, A. C. Lehum, J. R. Nascimento, A. Yu. Petrov and A. J. da Silva, Phys. Rev. D 87, 027701 (2013).

[16] A. C. Lehum, Phys. Rev. D 85, 125020 (2012).

[17] A. C. Lehum and A. J. da Silva, Phys. Lett. B 693, 393 (2010).

[18] L. Alvarez-Gaume, D. Z. Freedman, M. T. Grisaru, Report No. HUTMP 81/B111.

[19] A. F. Ferrari, E. A. Gallegos, M. Gomes, A. C. Lehum, J. R. Nascimento, A. Yu. Petrov and A. J. da Silva, Phys. Rev. D 82, 025002 (2010).

[20] I. L. Buchbinder, N. G. Pletnev and I. B. Samsonov, JHEP 1004, 124 (2010).

[21] I. L. Buchbinder, E. A. Ivanov, O. Lechtenfeld, N. G. Pletnev, I. B. Samsonov and B. M. Zupnik, JHEP 0910, 075 (2009).

[22] S. R. Coleman and E. Weinberg, Phys. Rev. D 7, 1888 (1973).

[23] A. Katz and Y. Shadmi, Phys. Rev. D 74, 115021 (2006) hep-ph/0605210.

[24] C. -k. Lee, K. -M. Lee and E. J. Weinberg, Phys. Lett. B 243, 105 (1990).

[25] A. C. Lehum, A. F. Ferrari, M. Gomes and A. J. da Silva, Phys. Rev. D 76, 105021 (2007).

[26] P. N. Tan, B. Tekin and Y. Hosotani, Phys. Lett. B 388, 611 (1996); Nucl. Phys. B 502, 483 (1997).

[27] A. G. Dias, M. Gomes and A. J. da Silva, Phys. Rev. D 69, 065011 (2004).

[28] R. Jackiw, Phys. Rev. D 9, 1686 (1974).

[29] A. F. Ferrari, M. Gomes, A. C. Lehum, J. R. Nascimento, A. Yu. Petrov, E. O. Silva and A. J. da Silva, Phys. Lett. B 678, 500 (2009).

[30] W. Siegel, Phys. Lett. B 84, 193 (1979).

[31] B. de Wit, M. T. Grisaru, M. Rocek, Phys. Lett. B 374, 297 (1996), hep-th/9601115; A. Pickering, P. 
West, Phys. Lett. B 383, 54 (1996), hep-th/9604147, M. T. Grisaru, M. Rocek, R. von Unge, Phys. Lett. B 383, 415 (1996), hep-th/9605149

[32] I. L. Buchbinder, S. M. Kuzenko, J. V. Yarevskaya, Nucl. Phys. B 411, 665 (1994), Phys. At. Nucl. 56, 680 (1993); I. L. Buchbinder, S. M. Kuzenko, A. Yu. Petrov, Phys. Lett. B 321, 372 (1994), Phys. At. Nucl. 59, 157 (1996).

[33] I. L. Buchbinder, M. Cvetic, A. Yu. Petrov, Mod. Phys. Lett. A 15, 783 (2000), hep-th/9903243 Nucl. Phys. B 571, 358 (2000), hep-th/9906141.

[34] F. S. Gama, M. Gomes, J. R. Nascimento, A. Yu. Petrov, A. J. da Silva, Phys. Rev. D84, 045001 (2011), arXiv: 1101.0724.

[35] D. Colladay, P. McDonald, Phys. Rev. D83, 025021 (2011), arXiv: 1010.1781.

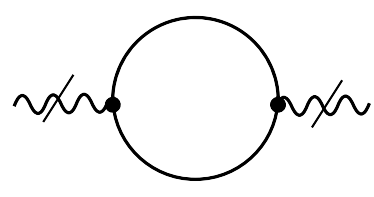

( a )

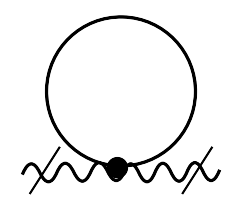

( b )

Figure 1: One-loop contributions to the gauge superfield effective action. Continuous lines represent the scalar superfield propagator, and wave crossed lines represent the external gauge superfield.
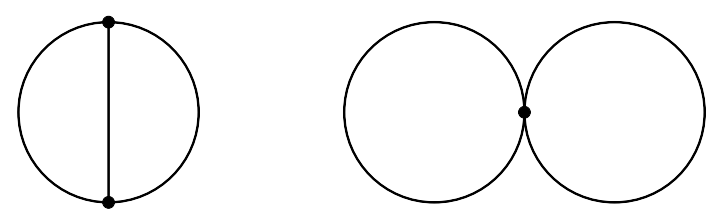

Figure 2: Topologies of two-loop diagrams that contribute to the Kählerian effective superpotential.
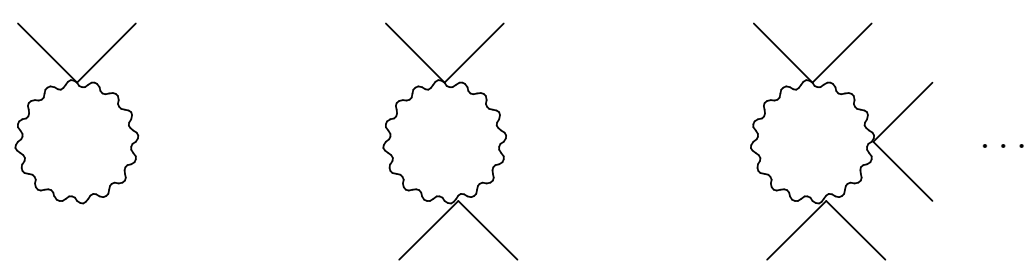

Figure 3: Supergraphs composed by gauge propagators only. 


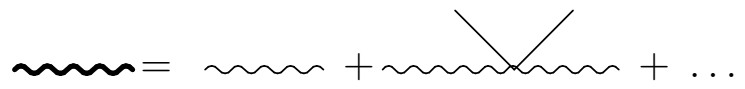

Figure 4: Dressed propagator.

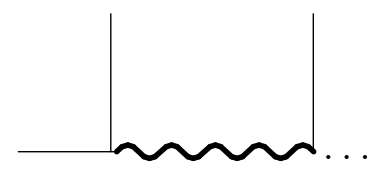

Figure 5: A link involving gauge and matter propagators.
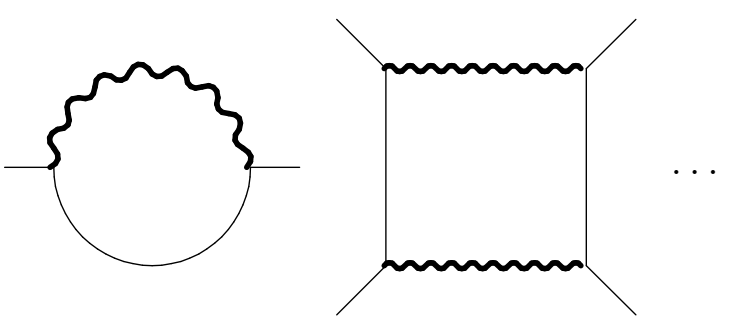

Figure 6: Supergraphs composed by gauge and matter propagators. 